\title{
A Novel Classification of the Internal Morphology of Mastoid Emissary Canals and st's Importance for Surgical Practice: an in Silico Anatomical Study
}

\author{
Olavo Barbosa de Oliveira-Neto, ${ }^{1,2}$ Juliana Haddad, ${ }^{3}$ Felippe Bevilacqua Prado, ${ }^{3}$ Ana Cláudia Rossi, ${ }^{3}$ Alexandre Rodrigues Freire ${ }^{3}$ \\ ${ }^{1}$ Institute of Biological and Health Sciences, Federal University of Alagoas, Maceió, AL, Brazil \\ ${ }^{2}$ CESMAC University Center, Maceió, AL, Brazil \\ ${ }^{3}$ Department of Morphology, Anatomy Division, Piracicaba Dental School, University of Campinas, Piracicaba, SP, Brazil
}

Disclose and conflicts of interest: none to be declared by all authors

\section{ABSTRACT}

Introduction: The aim of the present study was to determine the internal morphology of mastoid emissary canals and to classify them according to the number of external openings and the complexity of its morphology. In addition, we discussed the importance of the findings for surgical procedures performed in the retroauricular region.

Materials and Methods: An in silico anatomical study was performed using ninety-six dry human skulls, which were processed by computed tomography (CT) and underwent a segmentation process and construction of three-dimensional surfaces. The criteria used for classification of mastoid emissary canals was based on its morphological complexity and on the number of external openings.

Results: Canals were identified and initially classified into 4 groups. Groups were named after the number of external openings using numbers 1, 2, 3, and 4, which refer, respectively, to 1, 2, 3, or 4 foramens. Then, groups were divided into subgroups using up to the first three letters of the alphabet ( $a, b$, and $c$ ) according to the morphological complexity of mastoid emissary canals. Finally, 10 types of mastoid emissary canals were established, as follows: $1 a, 1 b ; 2 a, 2 b, 2 c ; 3 a$, $3 \mathrm{~b}, 3 \mathrm{c} ; 4 \mathrm{a}, 4 \mathrm{~b}$. We proposed a decreasing order of canals with potential to cause intracranial intercurrences and a decreasing order of canals with potential to cause moderate to severe hemorrhage.

Conclusion: A novel classification regarding the morphology of mastoid emissary canals was presented. In addition to the contributions in anatomical literature, it mainly represents new data to aid surgeons of various areas in the proper knowledge of important anatomical structures and planning of surgical procedures to reduce the occurrence of accidents and complications.

Keywords: Mastoid emissary canal; Mastoid emissary foramen; Segmentation; Classification; Computed tomography.

\section{Introduction}

The mastoid emissary foramen is a well addressed anatomical structure and it regards an opening located at the vicinities of the occipitomastoid suture. The mastoid emissary canal connects the mastoid emissary foramen to its internal opening, and through which passes the mastoid emissary vein, an important blood vessel that communicates the transverse or sigmoid sinus to the occipital or posterior auricular veins $^{1-8}$.

The mastoid emissary vein stablishes a communication that acts as an accessory drainage pathway to the internal jugular vein. Nevertheless, despite of what is classically known, authors had proven that the vertebral venous plexus (into which the mastoid emissary vein drains, indirectly) is the main pathway of cerebral venous drainage when one assumes an erect posture ${ }^{9-12}$. In addition, authors stated that this venous plexus enables a cooling mechanism for venous blood circulating through cephalic structures and prevents acute thermal damage $\mathrm{e}^{13,14}$.
Literature also cites that the knowledge regarding the anatomy of the mastoid emissary foramen and its canal is of utmost importance to perform surgical procedures in the mastoid region. Important surgical accidents and complications may occur due to injury to the mastoid emissary vein, such as hemorrhage, sigmoid sinus thrombosis, cerebellar ischemia and even death ${ }^{13,22}$.

Studies were performed to study the mastoid emissary canal. However, authors had been studying it with no technological resources that could enable a proper and precise anatomical description. Hence, its necessary to perform a new study with the proper tools to do so.

Thus, the aim of the present study was to determine the internal morphology of mastoid emissary canals and to classify them according to the number of external openings and the complexity of its morphology. In addition, we discussed the importance of the findings for surgical procedures in the retroauricular region. 


\section{Materials and Methods}

\section{Ethical approval}

The research project was developed a priori, which was assessed and approved by the institutional ethics committee (ID number: 13197519.8.0000.5418).

\section{Study design and sample}

An in silico anatomical study was performed at the institutional Morphology and Social Dentistry Departments (Piracicaba Dental School, University of Campinas, Brazil). Ninety-six dry human skulls were included for the research. Sample size calculation was performed after Keskil et al. 2003, which established the occurrence of the mastoid emissary foramen in $88,5 \%$ of dry human skulls ${ }^{5}$.

The present study included adult dry human skulls for analysis, with no distinction of sex or ancestralism. The following exclusion criteria were established: dry human skulls from non-adult individuals; skulls from a different species than Homo sapiens; skulls with any injury to the anatomical structures of interest; and hemi-skulls.

\section{Methods of morphological assessment}

The 96 adult dry human skulls were processed by computed tomography (CT) Aisteion Multislice 4 CT System (Toshiba Medical Systems Corporation - Japan), with $100 \mathrm{~mA}, 120 \mathrm{kV}$ and $1 \mathrm{~mm}$ voxel configuration. The CT images were imported to Materialise Mimics Research v.18 Software (Materialise, Belgium). In this software, a segmentation process of the mastoid emissary canal was performed, which consists in the identification, voxel demarcation, and isolation of this anatomical structure. For demarcation of the mastoid emissary canal, the voxels of bony structures were automatically selected by the grayscale threshold adjustment. Then, a manual demarcation of the mastoid emissary canal, including its external and internal openings, was performed using editing tools.

After segmentation, the three-dimensional surfaces were constructed, which comprises the segmentation of anatomical structures (already performed), and, the union and triangulation of voxels (Figure 1).

\section{Classification of mastoid emissary canals}

Using the three-dimensional reconstructions of mastoid emissary canals, we determined and characterized the different types mastoid emissary canals. Determination and characterization of canals were performed using visual inspection by two examiners (J.H., and O.B. de O.N.) with four years of experience in anatomical studies, each. These examiners scheduled weekly meetings to debate the anatomical findings to a third and more experienced examiner (A.R.F), with ten years of experience in anatomical and radiologic studies. The criteria used for classification of mastoid emissary canals was based on its morphological complexity and on the number of external openings (i.e. the number of mastoid emissary foramens).

\section{Results}

Mastoid emissary canals (MECs) were identified and initially classified into 4 groups. Groups were named after the number of external openings (i.e. the number of foramens) using numbers $1,2,3$, and 4 , which refer, respectively, to $1,2,3$, or 4 foramens. Then, groups were divided into subgroups using up to the first three letters of the alphabet (a,b, and c) according to the morphological complexity of mastoid emissary canals, where "a" would be the simplest and "c" would be the most complex. Finally, 10 types of mastoid emissary canals were established, as follows: 1a, 1b; 2a, 2b, 2c; 3a, $3 \mathrm{~b}, 3 \mathrm{c} ; 4 \mathrm{a}, 4 \mathrm{~b}$.

Types $1 \mathrm{a}$ and $1 \mathrm{~b}$ regard, respectively, one $\mathrm{C}$-shaped canal that arouses from one foramen and ends into one
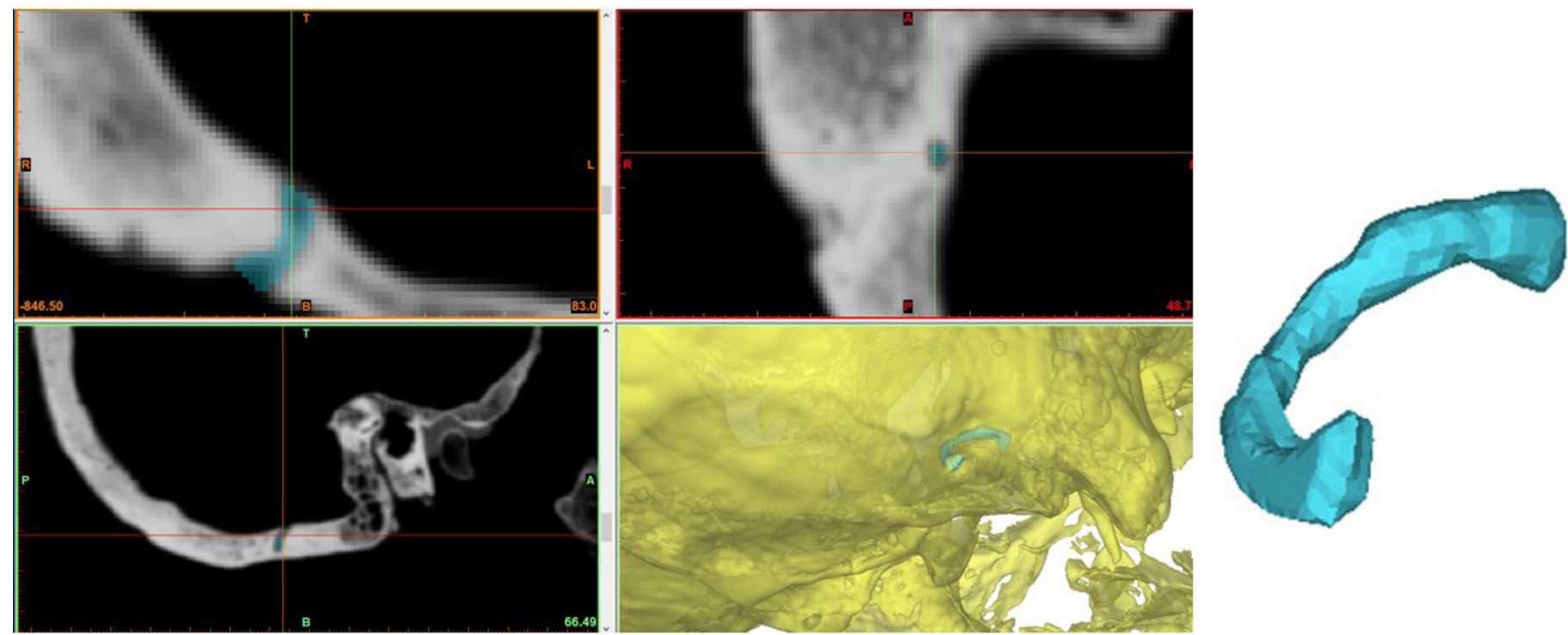

Figure 1. Steps performed to isolate the morphology of mastoid emissary canals. Segmentation (identification, voxel demarcation, and isolation) was performed prior to construction of three-dimensional surfaces. 
internal opening, and one S-shaped canal that arouses from one foramen and ends into one internal opening.

Type 2a is a bridge-like canal that only communicates the two mastoid emissary foramens and has no internal openings; type $2 \mathrm{~b}$ regards two independent canals arousing from individual foramens that converge into one another to form a single canal that ends in one internal opening; and type $2 \mathrm{c}$ was described as two individual canals arousing from individual foramens and that remain independent to end in two individual internal openings.

Regarding type 3 canals, type 3a was described as a combination of one canal that only communicates the two mastoid emissary foramens (a bridge-like canal, similar to type 2a) and has no internal openings associated to one canal that arouses from one foramen and ends into one internal opening (similar to type 1a); type $3 \mathrm{~b}$ comprised three independent canals that arouse from three individual foramens and converge into one another to form a single canal that ends into one internal opening; and type $3 \mathrm{c}$ was a combination of two independent canals arousing from individual foramens and converge into one another to form a single canal that ends in one internal opening associated to one canal that arouses from one foramen and ends into one internal opening.

The presence of four mastoid emissary foramens regarded the type 4 canals. Type 4 a was described as four foramens with independent canals that converge into one another to form a single canal that ends into one internal opening; and type $4 \mathrm{~b}$, the most complex of all, regarded two independent canals arousing from individual foramens that converge into one another to form a single canal that communicates transversally with one canal formed by the conversion of two independent canals that arouse from two individual foramens and converge into one another. The result of this union is one canal that ends into one internal opening.

Figures 2 and 3 show the types of canals from groups 1 and 2. Figures 4 and 5 show the types of canals from groups 3 and 4 . Table 1 presents the detailed parameters of classification and the morphological description of mastoid emissary canals found in the present study. a)

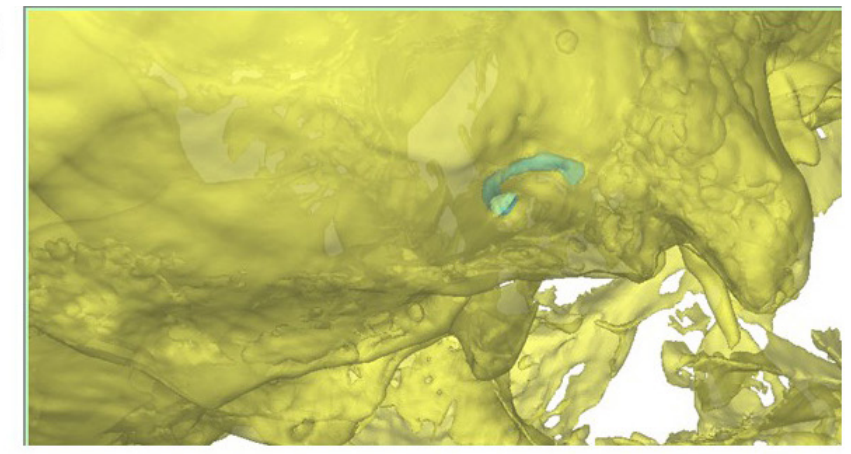

Type 1a

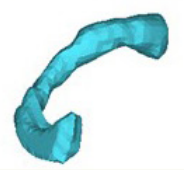

b)

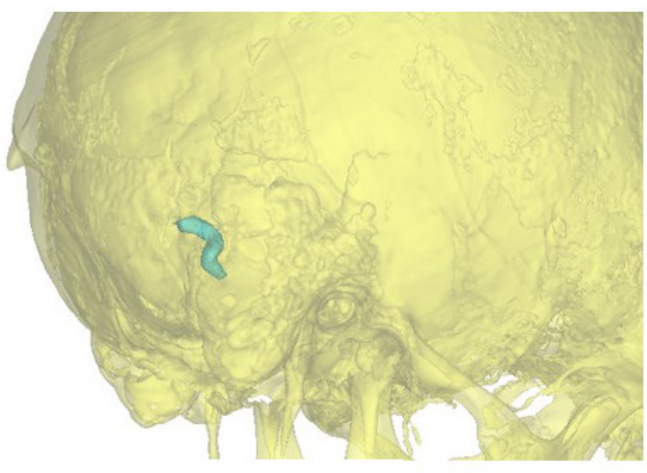

Type 1b

Figure 2. Mastoid emissary canals types 1a (a) and 1b (b).

a)

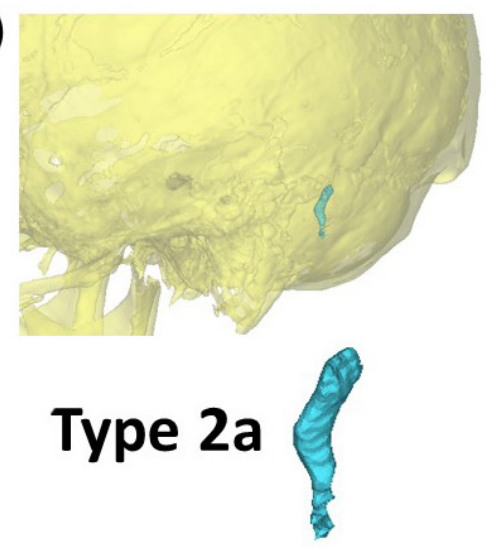

b)

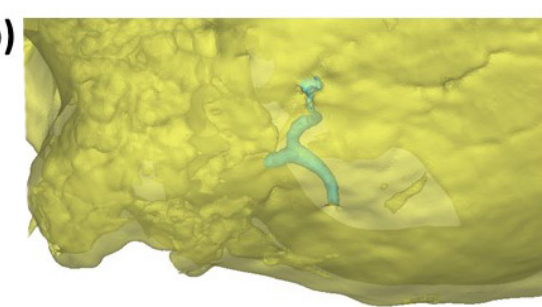

Type $2 b$ c)

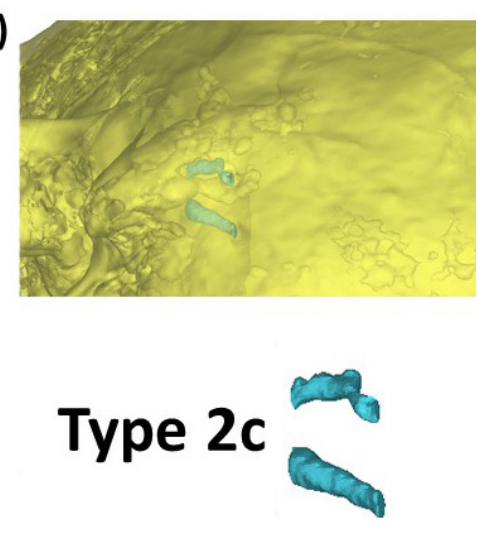

Figure 3. Mastoid emissary canals types 2a (a), 2b (b), and 2c (c). 
a)

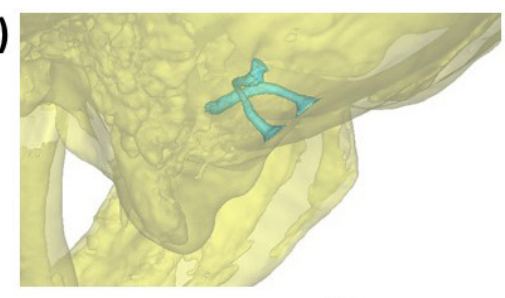

Type 3a

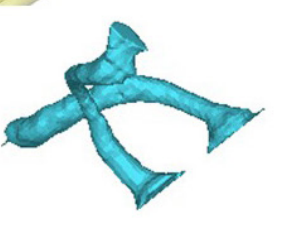

b)

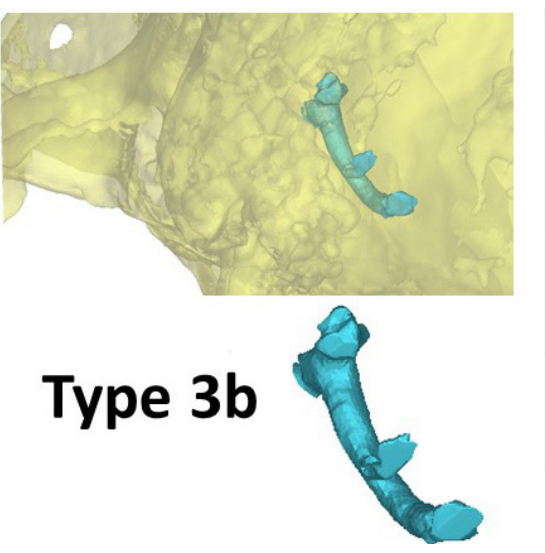

c)

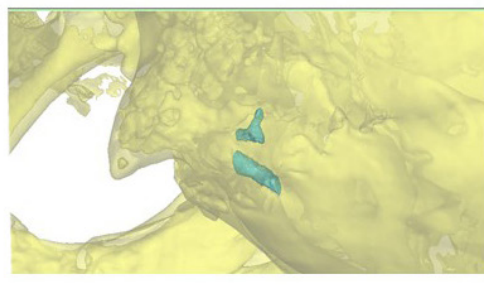

Type 3c

Figure 4. Mastoid emissary canals types $3 a(a), 3 b(b)$, and $3 c(c)$.

a)

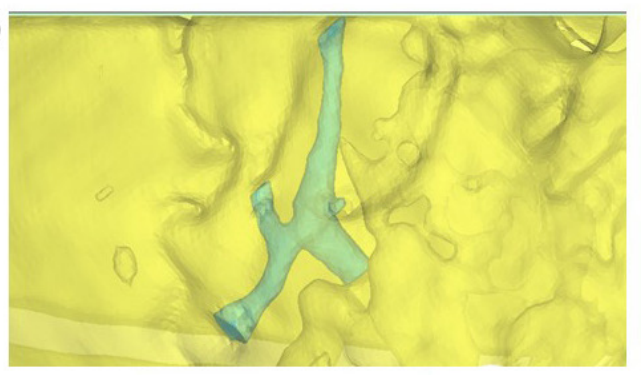

Type 4a

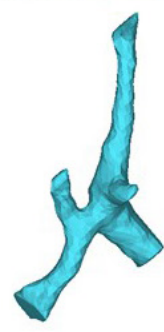

b)

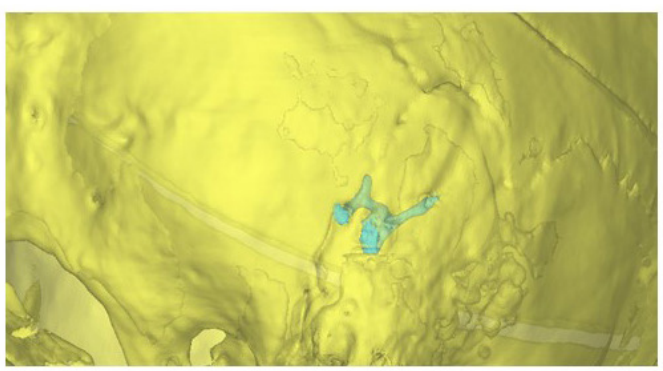

Type 4b

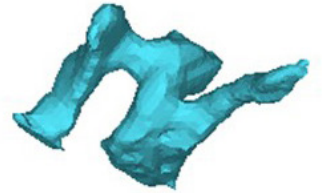

Figure 5. Mastoid emissary canals types $4 a(a)$ and $4 b(b)$

Table 1. Parameters used for classification of mastoid emissary canals regarding type, number of external openings (foramens), number of internal openings, and anatomical description.

\begin{tabular}{|c|c|c|c|}
\hline Type & $\begin{array}{l}\text { Number of } \\
\text { mastoid emissary } \\
\text { foramens }\end{array}$ & $\begin{array}{l}\text { Number of } \\
\text { internal } \\
\text { openings }\end{array}$ & Description of the canal \\
\hline $1 a$ & 1 & 1 & One C-shaped canal that arouses from one foramen and ends into one internal opening \\
\hline $1 \mathrm{~b}$ & 1 & 1 & One S-shaped canal that arouses from one foramen and ends into one internal opening \\
\hline $2 a$ & 2 & 0 & One bridge-like canal that only communicates the two mastoid emissary foramens and has no internal openings \\
\hline $2 b$ & 2 & 1 & $\begin{array}{l}\text { Two independent canals arousing from individual foramens that converge into one another to form a single canal } \\
\text { that ends in one internal opening }\end{array}$ \\
\hline 2c & 2 & 2 & $\begin{array}{l}\text { Two individual canals arousing from individual foramens and that remain independent to end in two individual } \\
\text { internal openings }\end{array}$ \\
\hline $3 a$ & 3 & 1 & $\begin{array}{l}\text { One canal that only communicates the two mastoid emissary foramens and has no internal openings }+ \text { One canal } \\
\text { that arouses from one foramen and ends into one internal opening }\end{array}$ \\
\hline $3 b$ & 3 & 2 & $\begin{array}{l}\text { Three independent canals that arouse from three individual foramens and converge into one another to form a } \\
\text { single canal that ends into one internal opening }\end{array}$ \\
\hline $3 c$ & 3 & 2 & $\begin{array}{l}\text { Two independent canals arousing from individual foramens and converge into one another to form a single canal } \\
\text { that ends in one internal opening + One canal that arouses from one foramen and ends into one internal opening }\end{array}$ \\
\hline $4 a$ & 4 & 1 & $\begin{array}{l}\text { Four foramens with independent canals that converge into one another to form a single canal that ends into one } \\
\text { internal opening }\end{array}$ \\
\hline $4 b$ & 4 & 1 & $\begin{array}{l}\text { Two independent canals arousing from individual foramens that converge into one another to form a single canal } \\
\text { that communicates transversally with one canal formed by the conversion of two independent canals that arouse } \\
\text { from two individual foramens and converge into one another. The result of this union is one canal that ends into } \\
\text { one internal opening }\end{array}$ \\
\hline
\end{tabular}




\section{Discussion}

The present study performed an in silico assessment of 96 adult dry human skulls using computational tools to determine the internal morphology of mastoid emissary canals and to classify them according to the number of external openings and the complexity of its morphology.

The types of mastoid emissary canals can be used for surgical planning to avoid accidents and complications in neurosurgery, otolaryngology surgery, and plastic and reconstructive surgery on the nuchal and posterior region of the scalp. Thus, the studied anatomical structure does not only present a descriptive anatomical importance, but also adds to clinical literature to aid healthcare professionals in the understanding and handling of adverse situations during surgeries, as well as to avoid them.

One must highlight that a wider perspective should be given to the mastoid emissary veins on surgical practice. Many surgeons state that, if injured, the mastoid emissary vein is not going to pose major problems because, if a moderate or a severe hemorrhage occur, the hemostasis of this vein is often easy to obtain, which is corroborated by us. However, professionals frequently forget or ignore that the problem often rests not in the mastoid emissary vein itself, but in the intracranial vein or veins that it communicates to, since the mastoid emissary vein is a potential pathway for thrombosis, thromboembolisms, and infections ${ }^{13-22}$. In this sense, noble intracranial structures, such as the cerebellum, the meninges, the brain stem, and the brain may be eventually put at jeopardy due to this communication, which corroborates and explains severe intercurrences regarding the injury of mastoid emissary veins during surgery, such as sigmoid sinus thrombosis, cerebellar ischemia, and even death ${ }^{23-26}$.

From a surgical perspective, despite one may think at a first glance, the simpler the mastoid emissary canal morphology is the higher is the chance of facing the aforementioned accidents and complications. Taking into considerations the concepts of collateral circulation $^{30,31}$, if a higher number of vessels are serving or draining a given anatomical structure, the lesser is the chance of this structure loses its blood supply if one or more vessels are injured or obstructed. Thus, a higher attention should be given if one is facing MECs of types 1a or $1 \mathrm{~b}$, because there is probably only one blood vessel that, in the retroauricular region, will communicate the exocranial and intracranial circulations. Moreover, we propose the following decreasing order of canals that will potentially create intracranial intercurrences for surgeons: $1 a>1 b>2 c>3 c>2 b>3 a>3 b>4 a>4 b>2 a$. Type 2a appears last because is a bridge-like canal with no internal openings. If the concern is the potential for moderate to severe hemorrhage in the surgical site, then the focus will be the number of (external) foramens, thus, we suggest the following decreasing order of canals with potential to cause this specific intercurrence: $4 b>4 a>3 c>3 b>3 a>2 c>2 b>2 a>1 b>1 a$.

To be best of our knowledge, the present study features a novel classification of mastoid emissary canals that has no previous papers to compare with. Thus, famous and well addressed classifications of anatomical structures other than the mastoid emissary canals were used to inspire authors regarding the names and numbers that would refer to the types of canals ${ }^{27-29}$.One must highlight that efforts were made to keep the classification as simple as possible in order to facilitate mnemonics, intraclass didactics, and communication between students, professors, researchers, and healthcare professionals.

The main strength of our study was to provide a novelty regarding the classification of an anatomical structure that can now be seen under a new morphological and functional perspective. Roser et al. 2016 and Demirpolat et al. 2016 studied mastoid emissary canals using routine CT images and multidetector computed tomography, respectively ${ }^{17-19}$. Although they considered biometrical parameters and performed statistical analysis, a 3D reconstruction of the canals was not performed and an attempt to classify the canals was not made. Several authors tried to study the mastoid canals using a metal wire ${ }^{1,3,4}$. However, this method is inadequate, and it does not provide the proper information to describe the morphology of mastoid canals.

The following difficulties occurred during the study and can be considered as limitations: 1) the overall quality of computed tomography impaired us from performing the segmentation of mastoid emissary canals in several antimeres; 2 ) for the same reason, a formal statistical analysis or even a simple descriptive statistics could not be performed because the number of viable canals was impaired for comparison.

In view of these difficulties, future researches should consider acquiring computed tomography of better quality (e.g. cone beam computed tomography), which would facilitate segmentation. Consequently, the use of computed tomography of better quality would also enable to perform a descriptive statistical synthesis or even a formal statistical analysis.

In conclusion, a novel classification regarding the morphology of mastoid emissary canals was presented. In addition to the contributions in anatomical literature, it mainly represents new data to aid surgeons of various areas in the proper knowledge of important anatomical structures and planning of surgical procedures to reduce the occurrence of accidents and complications.

\section{Acknowlegments}

We thank the Coordination for the Improvement of Higher Education Personnel (CAPES) of the Brazilian Ministry of Education in providing a Master of Science scholarship for the main author. 


\section{References}

1- Choudhry R, Raheja S, Gaur U, et al. Mastoid canals in adult human skulls. J Anat 1996;188, 217-219

2- Shaik HS, Shepur MP, Desai SD, et al. Study of mastoid canals and grooves in South Indian skulls. Indian J Med Healthc 2012; 1, 32-33

3- Singh M, Mishra A, Nagashima M. Mastoid Canals and Grooves in adult Japanese human skulls. J Anat Soc India 2004; 53(2), 40-43

4- Hussain SS, Muralidhar PS, Desai SD, et al. Study of Mastoid canals and grooves in South Indian skulls. IJMHC 2012; 1(1), 32-33

5- Keskil S, Gozil R, Calgunes E. Common surgical pitfalls in the skull. Surg Neurol 2003; 59(3), 228-231

6- Reis C, Desmuskh V, Zabramski JM et al. Anatomy of the mastoid emissary vein and venous system of the posterior neck region: neurosurgical implications. Neurosurg 2007; 61,193-201

7- Freire AR, Rossi AC, De Oliveira VCS et al. Emissary foramens of the human skull: Anatomical characteristics and its relations with clinical neurosurgery. Int J Morphol 2013; 31(1), 287-292

8- Gozil R, Kadioglu D, Calguner E. Occipital emissary foramen in skulls from Central Anatolia. Act Anat 1995; 153, 325-326

9- Falk D. Evolution of cranial blood drainage in hominids: enlarged occipital/marginal sinuses and emissary foramina. Am J Phys Anthropol 1996; 70, 311-324

10- Boyd GI. Emissary foramina of cranium in man and the anthropoids. J Anat 1930; 65,108-121

11- San Millan Ruiz D, Gailloud P, Rufenacht DA, et al. The craniocervical venous system in relation to cerebral venous drainage. Am J Neuroradiol 2002; 23,1500-1508

12- Pearce JMS. The craniospinal venous system. Eur Neurol 2006; 138(56),136-138

13- Valdueza JM, Von Munster T, Hoffman O et al. Postural dependency of the cerebral venous outflow. Lancet 2000; 355, 200-201

14- Kim LKP, Ahn CS, Fernandes AEL. Mastoid emissary vein: Anatomy and clinical relevance in plastic \& reconstructive surgery. Journal of Plastic, Reconstructive \& Aesthetic Surgery 2014; 67, 775-780 15- Irmak MK, Korkmax A, Erogul O. Selective brain cooling seems to be a mechanism leading to human craniofacial diversity observed in different geographical regions. Med Hypotheses 2004; 63, 974-979 16- Syed AZ, Sin C, Rios R, Mupparapu M. Incidental occurrence of an unusually large mastoid foramen on cone-beam computed tomography and review of the literature. Imaging Science in Dentistry 2016; 46, 39-45
17- Demirpolat G, Bulbul E, Yanik B. The prevalence and morphometric features of mastoid emissary vein on multidetector computed tomography. Folia Morphol 2016; 75(4): 448-453

18- Hampl M, Kacklik D, Kikalova K et al. Mastoid foramen, mastoid emissary vein and clinical implications in neurosurgery. Acta Neurochir 2018; 160(7),1473-1482

19- Roser F, Ebner FH, Ernemann U et al. Improved CT Imaging for Mastoid Emissary Vein Visualization Prior to Posterior Fossa Approaches. J Neurol Surg A Cent Eur Neurosurg 2016; 77(6), 511-514 20- Hoshi M, Yoshida K, Ogawa K et al. Hypoglossal neurinoma. Two case reports. Neurol Med Chir 2000; 40:489-493

21- Hadeishi H, Yasui N, Suzuki A. Mastoid canal and migrated bone wax in the sigmoid sinus: technical report. Neurosurgery 1995; 36(6), 1220-1224

22- Mortazavi MM, Tubbs RS, Riech S. Anatomy and Pathology of the Cranial Emissary. Neurosurgery 2012; 70,1312-1319

23-Louis RG, Loukas M, Wartmann CT et al. Clinical anatomy of the mastoid and occipital emissary veins in a large series. Surg Radiol Anat 2009; 31,139-144

24- Murlimanju BV, Chettiar GK, Prameela MD et al. Mastoid emissary foramina: an anatomical morphological study with discussion on their evolutionary and clinical implications. Anat Cell Biol 2014; 47, 202-206

25- Braga J, Boesch C. Further data about venous channels in South African Plio-Pleistocene hominids. J Hum Evol 1997; 33, 423-447

26- Hadimani GA, Bagoji IB. Study of Mastoid Canals and Grooves in North Karnataka Human Skulls. Journal of Clinical and Diagnostic Research 2013; 7(8), 1537-1539

27- Weine FS, Healey HJ, Gerstein H, et al. Canal configuration in the mesiobuccal root of the maxillary first molar and its endodontic significance. Oral surg 1969; 28, 419

28- Vertucci FJ. Root canal anatomy of the human permanent teeth. Oral Surg 1984; 58(5), 589-599

29- Tessier P. Anatomical Classification of Facial, Cranio-Facial and Latero-Facial Clefts. J max-fac surg 1976; 4, 69-92

30- Edwards EA. Scope and Limitations of Collateral Circulation. Arch Surg 1984; 119(7), 761-765

31- Faber JE, Chilian WM, Deindl E, et al. A Brief Etymology of the Collateral Circulation. Arteriosclerosis, Thrombosis, and Vascular Biology 2014; 34, 1854-1859

\section{Mini Curriculum and Author's Contribution}

1. Olavo Barbosa de Oliveira Neto - DDS; MSc; PhD student. Contribution: acquired data, performed formal analysis, and wrote the manuscript. ORCID: 0000-0003-1280-659X.

2. Juliana Haddad - MSc; PhD student. Contribution: assisted during data acquisition. ORCID: 0000-0001-6356-8726

3. Felippe Bevilacqua Prado - DDS, MSc, PhD. Contribution: reviewed and approved the first draft of the manuscript. ORCID: 0000-0001-5636-6915.

4.Ana Cláudia Rossi - DDS; PhD. Contribution: provided insights to the methodology. ORCID: 0000-0002-8718-4898

5. Alexandre Rodrigues Freire - DDS; MSc; PhD. Contribution: reviewed and approved the final version of the manuscript. ORCID: 0000-0002-0175-0896.

Received: June 15, 2021

Accepted: August 8, 2021
Corresponding author

Olavo Barbosa de Oliveira Neto

E-mail: olavobarbosa91@gmail.com 